\title{
Unexpected softness of bilayer graphene and softening of A-A stacked graphene layers
}

\author{
Y. W. Sun $\odot,{ }^{1, *}$ D. Holec,,${ }^{2, \dagger}$ D. Gehringer, ${ }^{2}$ O. Fenwick, ${ }^{1}$ D. J. Dunstan $\odot,{ }^{3}$ and C. J. Humphreys ${ }^{1, \ddagger}$ \\ ${ }^{1}$ School of Engineering and Materials Science, Queen Mary University of London, London E1 4NS, United Kingdom \\ ${ }^{2}$ Department of Materials Science, Montanuniversität Leoben, Leoben 8700, Austria \\ ${ }^{3}$ School of Physics and Astronomy, Queen Mary University of London, London E1 4NS, United Kingdom
}

(Received 9 October 2019; revised manuscript received 13 February 2020; accepted 2 March 2020; published 20 March 2020)

\begin{abstract}
Density functional theory has been used to investigate the behavior of the $\pi$ electrons in bilayer graphene and graphite under compression along the $c$ axis. We have studied both conventional Bernal (A-B) and A-A stackings of the graphene layers. In bilayer graphene, only about $0.5 \%$ of the $\pi$-electron density is squeezed through the $s p^{2}$ network for a compression of $20 \%$, regardless of the stacking order. However, this has a major effect, resulting in bilayer graphene being about six times softer than graphite along the $c$ axis. Under compression along the $c$ axis, the heavily deformed electron orbitals (mainly those of the $\pi$ electrons) increase the interlayer interaction between the graphene layers as expected, but, surprisingly, to a similar extent for A-A and Bernal stackings. On the other hand, this compression shifts the in-plane phonon frequencies of A-A stacked graphene layers significantly and very differently from the Bernal stacked layers. We attribute these results to some $s p^{2}$ electrons in A-A stacking escaping the graphene plane and filling lower charge-density regions when under compression, hence, resulting in a nonmonotonic change in the $s p^{2}$-bond stiffness.
\end{abstract}

DOI: 10.1103/PhysRevB.101.125421

\section{INTRODUCTION}

Graphene has many extraordinary properties, such as its large in-plane stiffness [1], mainly due to its $s p^{2}$ covalent bond network. However, in multilayer graphene, it is the $\pi$-electron orbitals that determine the interlayer interaction. Each number of layers, and each stacking order, A-A and conventional A-B (Bernal), has distinct properties. It is of fundamental importance and interesting to understand and quantify how the $s p^{2}$ - and $\pi$-electron orbitals affect each other. In this paper, we employ density functional theory (DFT) to study the change in the in-plane properties under uniaxial compression along the $c$ axis and the effect of the stacking order, which we expect to have a significant impact on the behavior of the $\pi$ electrons.

Graphene is a two-dimensional (2D) material, but it has three-dimensional (3D) properties, such as the out-of-plane stiffness, as its $\pi$ orbitals resist out-of-plane compression. In our previous work, we experimentally applied hydrostatic pressure to monolayer graphene, measured the shift of its in-plane phonon frequency with pressure, and quantified the out-of-plane stiffness from this shift. We found a similar outof-plane stiffness of monolayer graphene $(1.4 \pm 295 \mathrm{GPa})$ to graphite $(33.8 \pm 3.0 \mathrm{GPa}$ [2]) within experimental error [3]. In the present theoretical paper, the computational results show a completely unexpected six times smaller out-of-plane stiffness of bilayer graphene than graphite. We relate this to the asymmetric distribution of $\pi$ orbitals on each side of a graphene layer in a bilayer (the distribution is symmetric in

\footnotetext{
*yiwei.sun@qmul.ac.uk

†david.holec@unileoben.ac.at

¥c.humphreys@qmul.ac.uk
}

monolayer and graphite), owing to the possibility of the $\pi$ electron density being compressed through the $s p^{2}$ network.

In addition, 2D analysis is usually applied to graphene due to its large elastic anisotropy [4]. In particular, the frequencies of the in-plane phonons of graphene bilayers have been related to only the in-plane strain [5-8], despite the out-of-plane strain being about 30 times larger than the in-plane strain under hydrostatic compression. In our previous work, we quantified the contribution of the out-of-plane strain to the in-plane phonon frequency and found that it could not be neglected. We attributed this contribution to the compression of the $\pi$ electrons into the $s p^{2}$ network to alter the in-plane bond [9]. To further understand this behavior, we investigate the effect of the stacking order. The stacking order has a considerable impact on the properties of graphene bilayers. We take the A-A stacking as an extreme example to compare with the normal A-B Bernal stacking. A-A stacked graphene layers are expected to have larger equilibrium interlayer separations and higher energy than Bernal stacking [10]. They have some unique electronic and magnetoelectronic properties [11], such as good tunneling conductance [12] and Fano antiresonance in the conductance [13]. They also have high optical conductivity in the terahertz range [14]. Although most studies are theoretical, A-A stacked graphene layers have also been experimentally observed. Lauffer et al. observed an area of A-A stacking in bilayer graphene by scanning tunneling microscopy and spectroscopy [15], and Liu et al. found AA stacked bilayer graphene close to the folding edge and concluded that the A-A stacking minimized the local strain during heat treatment [16]. In a theoretical work, de Andres et al. reported an interlayer covalent bond after compressing A-A stacked bilayer graphene to a separation of $0.156 \mathrm{~nm}$ [17]. This is a very large compression, far beyond the smallest 
interlayer spacing of about $0.23 \mathrm{~nm}$ considered in this paper. We chose the range of compression to focus on the behavior of the $\pi$ electrons before the $s p^{2}$ to $s p^{3}$ transition. We find a six times softer bilayer graphene than graphite out-of-plane for both Bernal and A-A stackings. Also, unexpectedly, there is a significant difference between Bernal and A-A stackings in the response of in-plane stiffness to out-of-plane compression for both bilayer graphene and graphite.

\section{METHODS}

We employed density functional theory (DFT) $[18,19]$ as implemented in the Vienna $A b$ initio Simulation Package (VASP) [20] to study bilayer graphene and graphite with A$A$ and Bernal stackings at $0 \mathrm{~K}$. We treated the exchangecorrelation effects by the generalized gradient approximation as parametrized by Perdew et al. [21] and used the projector augmented-wave method pseudopotentials [22] for carbon. We used the plane-wave cutoff energy of $900 \mathrm{eV}$ and sampled the reciprocal unit cell with an $18 \times 18 \times 9 k$ mesh to achieve the optimized accuracy of the results. We included the effects of van der Waals (vdW) interactions using the Grimme method [23] as implemented in the VASP code. Nevertheless, estimating the vdW force accurately is challenging in DFT. As a result, we found the equilibrium interlayer distance of 3.20 $\AA$ for Bernal stacked graphite, compared to the experimental value at equilibrium of $3.35 \AA$ [2]. We calculated the vibrational frequencies at the Brillouin-zone center, the $\Gamma$ point using a $2 \times 2 \times 2$ supercell employing the finite displacement method as implemented in the PHONOPY code [24]. In this paper, we are mainly interested in the shift of the phonon frequency with the change in atomic positions, which is a measure of the anharmonicity of bonding, that would only be weakly affected by the imperfectly estimated vdW force. The structural model and charge density were visualized using the VESTA software [25].

\section{RESULTS AND DISCUSSIONS}

\section{A. Out-of-plane stiffness}

The out-of-plane stiffness of bilayer graphene and graphite is mainly determined by the overlap of $\pi$ orbitals of neighboring graphene layers. We applied uniaxial stress along the $c$ axis (where the in-plane stress is 0) to the A-A and Bernal stacked bilayer graphene and graphite. We varied the interlayer distance, optimized the in-plane lattice parameters at each interlayer distance and then calculated the uniaxial stress and the properties of graphene layers at this geometry. Uniaxial stress is plotted against interlayer distance in Fig. 1.

We fit the data of the uniaxial stress to the interlayer distance by the one-dimensional (along the $c$ axis) analog of the Murnaghan equation [2],

$$
a_{33} / a_{33_{0}}=\left[\left(c_{33}^{\prime} / c_{33}\right) P+1\right]^{-1 / c_{33}^{\prime}},
$$

where $a_{33_{0}}$ is the unstrained interlayer distance, $P$ is the uniaxial stress, $c_{33}$ is the elastic constant at equilibrium $c_{33_{0}}$, and $c_{33}^{\prime}$ is the rate of change of $c_{33}$ with stress. The Murnaghan equation is derived from the equations of continuum mechanics and expresses the fact, here, that the more graphene layers are compressed, the more difficult it is to compress

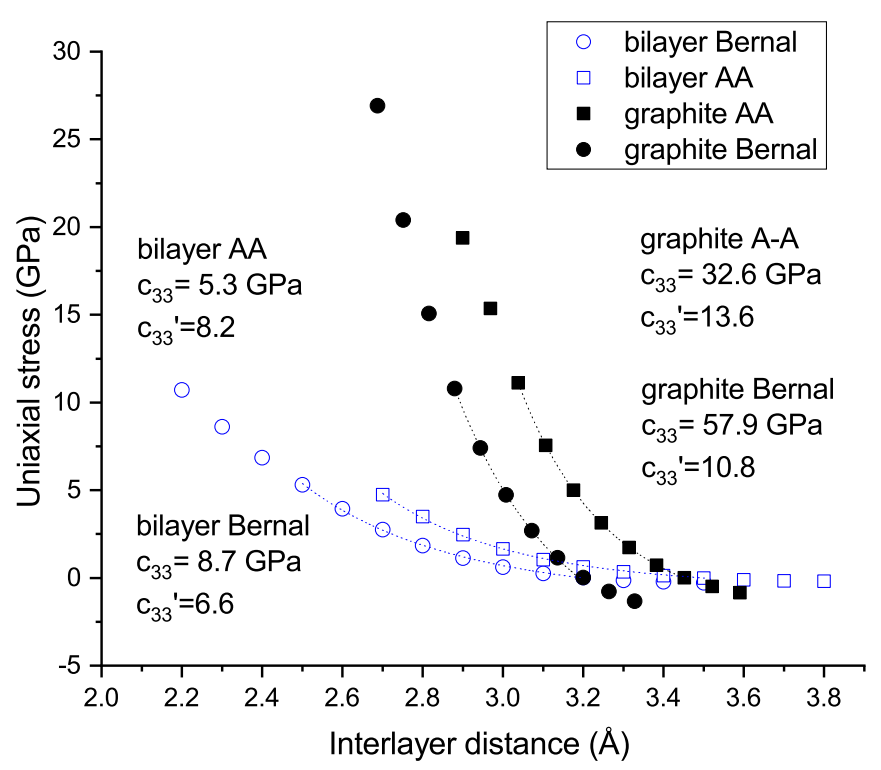

FIG. 1. The uniaxial stress along the $c$ axis applied to A-A and Bernal stacked bilayer graphene and graphite is plotted with the corresponding interlayer distance at which the stress was calculated. The black solid points are for graphite, and the blue open circles are for bilayer graphene. Circles are for Bernal stacking, and squares are for A-A. The data were fitted by Eq. (1), and the fitting results are presented.

them further [26]. This applies to the $c$ axis, and so the one-dimensional analog is realistic when we focus on the out-of-plane stiffness [2]. Essentially, this version of the Murnaghan equation is empirical, consisting of only two fitting parameters, the modulus of incompressibility, and its first derivative to pressure. The good fit shown in Fig. 1 confirms its applicability, and a more complicated equation of state with more fitting parameters would be likely to overfit the data. For bilayer graphene, we obtained $c_{33}=5.3 \mathrm{GPa}$ and $c_{33}^{\prime}=8.2$ for A-A stacking at DFT equilibrium $a_{33_{0}}=3.50 \AA, c_{33}=$ $8.7 \mathrm{GPa}$ and $c_{33}^{\prime}=6.6$ for Bernal stacking at $a_{33_{0}}=3.20 \AA$. For graphite, we obtained $c_{33}=32.6 \mathrm{GPa}$ and $c_{33}^{\prime}=13.6$ for A-A stacking at $a_{33_{0}}=3.45 \AA$ and $c_{33}=57.9 \mathrm{GPa}$ and $c_{33}^{\prime}=10.8$ for Bernal stacking at $a_{33_{0}}=3.20 \AA$, compared to the experimental value $c_{33}=38.7 \pm 0.7 \mathrm{GPa}$ [4] and $c_{33}^{\prime}=$ $11.8 \pm 0.9$ at the experimental equilibrium distance $a_{33_{0}}=$ $3.35 \AA$ ̊ [2] for Bernal stacked graphite. DFT evaluates Pauli repulsion more accurately than the vdW attraction, which suggests focusing on the data in the "DFT compression" region (where the calculated stress is positive), which, indeed, is where we are interested. We, therefore, fitted over zero stress. The fitted values of the parameters in the Murnaghan equation may depend on the pressure range of the fitting (although weakly as the fittings are good as shown). We, therefore, fit the data over the same pressure range for Bernal and A-A stackings. We also bear in mind that the interlayer distance is the input in the calculations. The calculated value of the elastic constant $c_{33}$ does depend on the value of $a_{33_{0}}$, and a smaller value used in the fit of $a_{330}$ is obtained by DFT than from experiments. For the Bernal stacked bilayer graphene, the $c_{33}$ is $8.7 \mathrm{GPa}$ at $a_{33}=3.20 \AA$ (DFT 0 stress). As the experimental $a_{33}$ of graphite at equilibrium is larger 
than the DFT bilayer value, the DFT $c_{33}$ at the experimental equilibrium $a_{33}=3.35 \AA$ is smaller than $8.7 \mathrm{GPa}$ (further away from the values for graphite). The calculated out-ofplane stiffness of graphite of both stacking orders is generally consistent with the experimental values of Bernal stacked graphite, whereas the $c_{33}$ of bilayer graphene is about six to seven times smaller. Bilayer graphene is very soft along the $c$ axis, much softer even than graphite. The stacking order does make a slight difference, and, as expected, the A-A stacking is softer out-of-plane initially than the Bernal stacking due to the larger equilibrium interlayer spacing but grows stiffer faster under compression as the $\pi$-electron orbitals from the neighboring layers are in contact all around the carbon hexagons. The smooth fit by the Murnaghan equation confirms that there is no $s p^{2}$ to $s p^{3}$ transition over the stress range used. We note that large structural change might be prohibited by the small unit-cell setup (a complete $s p^{2}$ to $s p^{3}$ transition might not be allowed in this simulation, but we are anyway not above $30 \mathrm{GPa}$, which is usually needed for a rapid conversion of graphite to diamond [27]). What is important here is that the following observations in this paper are not a result of the rehybridization of $s p^{2}$ bonding to $s p^{3}$.

\section{B. Compressing the electron density under uniaxial stress}

An important difference between bilayer graphene and graphite is the asymmetric electron distribution on each side of a graphene layer in a bilayer, which could be the cause for the dramatic difference in the out-of-plane stiffness between them. We investigated how much the $\pi$-electron density can be squeezed through the $s p^{2}$ network under compression due to the asymmetric distribution. We modeled bilayer graphene with A-A and Bernal stackings, varied the interlayer spacing, and integrated the charge between the two graphene layers of the electrons in the outmost occupied shell. In our previous work [3], we realized that "graphene is thin, but not infinitely so" [28]. In the present paper, we set infinitely thin boundaries across the center of all the carbon atom nuclei of a graphene layer and integrated the charge between these boundaries. We do not deliberately distinguish $s p^{2}$ - from $\pi$-electron densities because it is reasonable to think that, if electron density can be squeezed through a graphene plane, then, it would be the $\pi$-electron density, which is highly compressed. In addition, if $s p^{2}$-electron density is squeezed through a graphene plane due to the compression of $\pi$ orbitals, this might affect the compressibility of graphene layers as well.

For both stacking orders, we had four carbon atoms in a unit cell and $16 e$ was the sum of the valence charge from between and outside the two layers. We plot the integrated valence charge density between the layers versus the interlayer distance in Fig. 2. Compared to Bernal stacking, the optimized interlayer spacing for A-A stacking is larger, and it is harder for the electron density to be compressed through the $s p^{2}$ network as expected ( $\pi$ orbitals of all the carbon atoms have another carbon atom directly above or below in an A-A stacked bilayer). Nevertheless, for both stacking orders, the absolute amount of electron charge density compressed through is extremely small. Only $0.53 \%$ of the valence charge density in between the graphene bilayers is squeezed out under a compression of $23 \%$ reduction in volume in

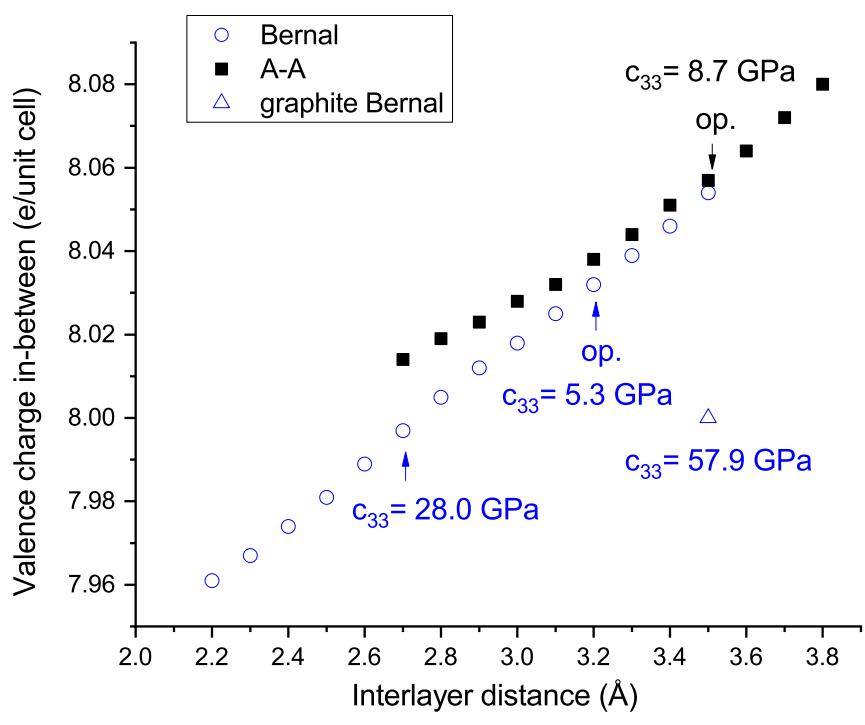

FIG. 2. The integrated valence charge between the two graphene layers is plotted versus the interlayer distance of the bilayer graphene for A-A (blue open circles) and Bernal stacking (black solid squares). The interlayer distance at equilibrium for each stacking is arrowed. We also plot the calculated equilibrium spacing for Bernal stacked graphite (blue open triangle). The values of $c_{33}$ from the fittings in Fig. 1 at a few key points are presented. The difference between Bernal stacked bilayer graphene and graphite for the elastic constant $c_{33}$ when both have $8-e /$ unit cell valence charge between the graphene bilayers, indicates that the $c_{33}$ is not entirely determined by the amount of interlayer electronic charge.

the A-A stacking and under a similar compression of $22 \%$ in the Bernal stacking, only $0.63 \%$ of the valence charge density is compressed out. The consequential large increase in the charge density between the graphene bilayers under compression indicates a large deformation or overlap of the electron orbitals (mainly of the $\pi$ electrons one would expect). This validates the interpretation in our previous work that the compression of the $\pi$-electron orbitals into the $s p^{2}$ network is responsible for the significant contribution of the out-of-plane strain to the frequency shifts of the in-plane phonons [9]. There is no transition of $s p^{2}$ towards $s p^{3}$, the sign of which is that carbon atoms go out of the $s p^{2}$ plane. We optimized the geometry at each interlayer separation and did not observe any disruption of the $s p^{2}$ plane.

We also arrowed on Fig. 2 the elastic constant $c_{33}$ of bilayer graphene and Bernal stacked graphite at equilibrium, from the fittings presented in Fig. 1 (where the dashed lines start). The valence charge density between graphene bilayers is always $8 e /$ unit cell in graphite due to the symmetry of the system. The $c_{33}$ of bilayer graphene is still only about half that of graphite when the interlayer valence charge density is compressed so that the interlayer valence charge is 8 . Therefore, the difference in the out-of-plane stiffness between bilayer graphene and graphite is not due to the different interlayer valence charge densities. It is also not due to the different interlayer distances at equilibrium as shown in the last section. It is reasonable to think that the asymmetric distribution of electrons on each side of a graphene layer in bilayer graphene is responsible. More precisely, the ultrasoftness of bilayer 
graphene is because the $\pi$-electron density can be compressed out of the interlayer. Nevertheless, bilayer graphene being six times softer than graphite is, indeed, unexpected and intriguing.

\section{Phonons of graphite under compression}

We now move on from the out-of-plane stiffness to the in-plane stiffness of graphene layers. We investigated how the stacking orders affect the in-plane stiffness under outof-plane compression. We modeled A-A and Bernal stacked graphite under hydrostatic pressure with the equilibrium lattice parameter $a_{33}$ of 3.50 and $3.20 \AA$, respectively. We applied pressure by setting a smaller 3D unit-cell volume, optimizing the geometry and calculating the corresponding pressure. We plot the calculated hydrostatic pressure versus the unit-cell volume to obtain bulk moduli for A-A and Bernal stacked graphite in Fig. 3(a). We fitted the data using the Murnaghan equation [26] and obtained the unstrained bulk modulus $B_{0}=$ $30.5 \mathrm{GPa}$ and its shift rate with pressure $B^{\prime}=11.2$ for A-A stacking and $B=45.1 \mathrm{GPa}$ and $B^{\prime}=10.4$ for Bernal stacking, close to the published experimental values for Bernal stacked graphite of $B=33.8 \pm 3.0 \mathrm{GPa}$ and $B^{\prime}=8.9 \pm 1.0$ [2] (the small discrepancy is due to the estimated equilibrium $a_{33}$ using DFT, depending on the vdW).

The small bulk modulus of graphite is due to its weak interlayer interaction of which the frequency of the layer breathing mode (LBM) is a good indicator. We plot the LBM frequency versus pressure in Fig. 3(b) and empirically fit the data by [2]

$$
\omega(P) / \omega_{0}=\left[\left(\delta_{0} / \delta^{\prime}\right) P+1\right]^{\delta^{\prime}},
$$

where $\omega$ is the frequency, $\delta_{0}$ is the logarithmic pressure derivative $(d \ln \omega / d P)_{P=0}$, and $\delta^{\prime}$ is the pressure derivative of $d \ln \omega / d P$, accounting for the curvature. We obtained the values of the fitting parameters of $\delta_{0}=0.29 \mathrm{GPa}^{-1}$ and $\delta^{\prime}=$ 0.40 for A-A stacked graphite and $\delta_{0}=0.18 \mathrm{GPa}^{-1}$ and $\delta^{\prime}=$ 0.37 for Bernal stacking with the latter being comparable to the experimental values of $\delta_{0}=0.15 \mathrm{GPa}^{-1}$ ( $\delta^{\prime}$ not available) [29].

For the bulk modulus and the LBM frequency of Bernal stacked graphite under hydrostatic pressure, the theoretical results are very close to those from experiments, validating the calculations in this paper. The difference in stacking order only makes a marginal difference (compared to the difference between bilayer graphene and graphite and the difference in the in-plane stiffness, which will be presented later) in the outof-plane stiffness and the interlayer interaction. This is mainly determined by the overlap of the $\pi$-electron orbitals where the impact of the stacking order ought to be large. Again, no $s p^{2}$ to $s p^{3}$ transition occurs in the plotted pressure range. The good agreement on the $B^{\prime}$ and the $\delta_{0}$ for the LBM between the experimental values in the literature and our calculations indicates the validity of the computational results from our modeling.

We now focus on the effect of the compression on the in-plane stiffness of graphene layers. The frequencies of the four in-plane phonons of A-A stacked graphite are presented in Fig. 3(c)-two carbon atoms vibrate in-line antiphase along longitudinal or transverse directions in the hexagonal

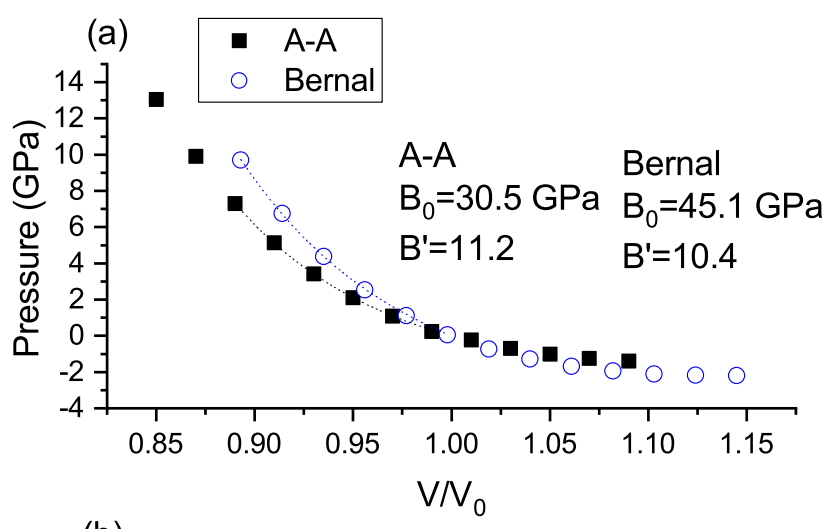

(b)
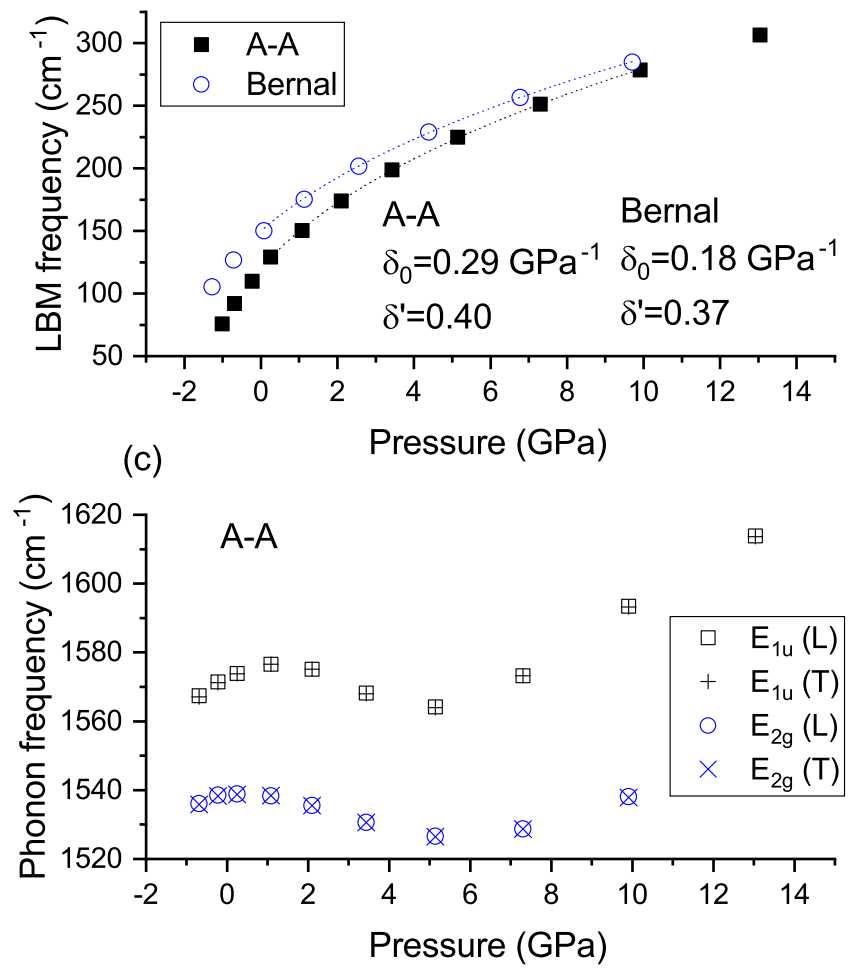

FIG. 3. Hydrostatic pressure was applied to A-A and Bernal stacked graphite. (a) The calculated pressure is plotted versus the ratio of the unit-cell volume to the unstrained volume (A-A stacking in black solid squares and Bernal stacking in blue open circles). Fitting using the Murnaghan equation is shown as dashed lines, black for A-A stacking and blue for Bernal stacking. (b) The frequencies of the LBM for A-A and Bernal stacked graphite are plotted versus hydrostatic pressure (A-A stacking in black solid squares and Bernal stacking in blue open circles). The data are fitted using Eq. (2) as dashed lines, black for A-A stacking and blue for Bernal stacking. (c) The frequencies of the four in-plane phonon modes (as labeled) for A-A stacked graphite are plotted versus pressure. We use the same notation to label the four phonon modes as for Bernal stacked graphite to be consistent, despite the symmetry being different. $L$ is for longitudinal modes, and $T$ is for transverse modes.

plane of graphene (labeled as $L$ and $T$ ), and the vibrations in the two layers vibrate in or out of phase (called $E_{1 u}$ and $E_{2 g}$ in Bernal stacking, we use these labels for A-A stacking too; illustration diagram in Ref. [30]). The vibrational frequencies of the $L$ and $T$ modes are degenerate as expected. The 
frequencies of both $E_{1 u}$ and $E_{2 g}$ in the A-A stacked graphite shift nonmonotonically with pressure, unlike in the Bernal stacking (where the frequencies increase almost linearly with pressure [9]), over the pressure range where no $s p^{2}$ to $s p^{3}$ transition occurs. The compression of the $\pi$-electron orbitals not only modifies the shift rates of the in-plane phonons with pressure in Bernal stacked graphite, but also changes the sign of the shifts in A-A stacking.

We excluded the effect of in-plane strain by applying uniaxial stress along the $c$ axis to graphite of each stacking order. The interlayer distance was the input in the calculations. We calculated the corresponding uniaxial stress at each interlayer distance.

We again calculated the LBM frequencies as a measure of interlayer interaction and plot them versus the interlayer distance in Fig. 4(a). We fit the data by [31]

$$
\omega(P) / \omega_{0}=\left[r(P) / r_{0}\right]^{-3 \gamma},
$$

where $\omega$ is the frequency of the LBM and $r$ is the interlayer distance. We obtained $\gamma=2.30$ for A-A stacked graphite, compared with the published $\gamma=2.36$ for Bernal stacked graphite [31]. The result indicates that the interlayer interaction of A-A stacked graphite increases at nearly the same rate as that of Bernal stacked graphite under uniaxial stress.

Under uniaxial compression along the $c$ axis, the stacking order of graphite has only a small impact on the out-of-plane stiffness and the interlayer interaction. We then investigated the in-plane properties. We calculated the frequencies of the four in-plane phonons for A-A stacked graphite at each interlayer distance and plot the data in Fig. 4(b). We note that the out-of-plane compression has a large impact on the in-plane phonons not only shifting the frequencies significantly, but also changing the sign of the shift of the in-phase vibrations. On the other hand, Bernal stacked graphite behaves more reasonably in that the frequencies of the in-phase modes $\left(E_{1 u}\right)$ increase whereas the out-of-phase modes $\left(E_{2 g}\right)$ decrease with increasing interlayer coupling [9]. We would like to point out again that no $s p^{2}$ to $s p^{3}$ transition occurs over the pressure range used.

We present the shift of in-plane phonon frequencies with interlayer distance for A-A- and Bernal stacked bilayer graphene under uniaxial compression along the $c$ axis in the Supplemental Material [32]. Similar to graphite, the frequency shifts of three out of four phonon modes of the A-A stacking changes sign twice over a small pressure range to $5 \mathrm{GPa}$, whereas the phonon frequency of the Bernal stacking shifts more uniformly, indicating that the substantial impact of the out-of-plane compression on the in-plane phonons largely depends on the stacking orders. An interpretation is provided in the next subsection.

\section{Change in charge density under uniaxial strain}

Both A-A- and Bernal stacked graphene bilayers are very soft to compress along the $c$ axis, and under compression, small amounts of electron charge density are squeezed through the $s p^{2}$ network (although this might be the cause of the huge difference between bilayer graphene and graphite). Our finding that the stacking order has very small effect on the out-of-plane stiffness, and the interlayer interaction suggests

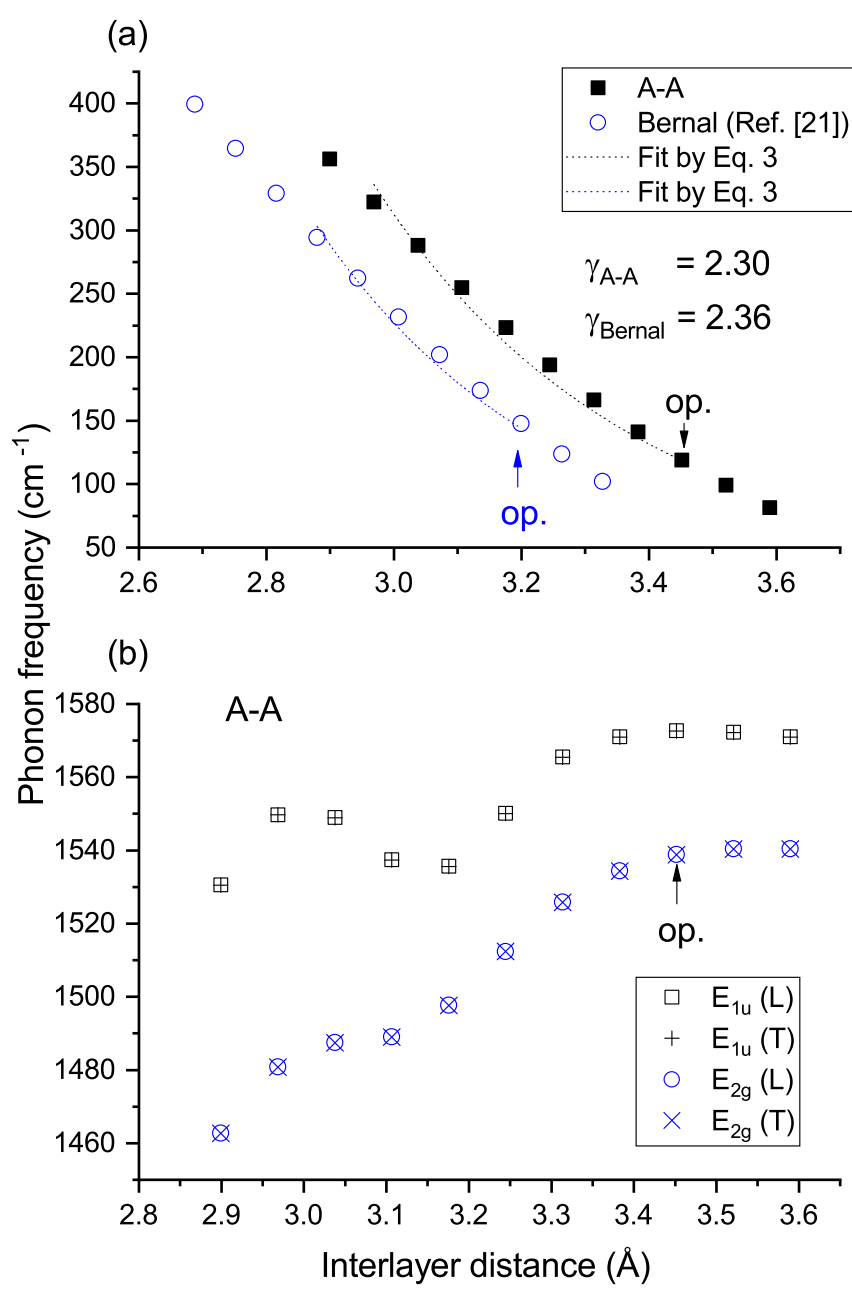

FIG. 4. Uniaxial stress along the $c$ axis was applied to A-A and Bernal stacked graphite. (a) The frequency of the LBM for A-A stacked graphite (black solid squares) is plotted versus the interlayer distance. The data are fitted by Eq. (3) in dashed lines. The published data and the fit for Bernal stacked graphite is presented for comparison [31]. (b) The frequencies of the four in-plane phonon modes (as labeled) for A-A stacked graphite are plotted versus the interlayer distance under uniaxial stress. We use the same notation to label the phonon modes as those four in Bernal stacked graphite to be consistent, despite the symmetry being different. $L$ is for the longitudinal modes, and $T$ is for the transverse modes.

that the overlap of $\pi$ orbitals of neighboring layers is nearly the same for Bernal and A-A stackings, probably because the electrons fill the area near the carbon hexagon center of low electron density. Plausibly, it is the $\pi$ electrons that do the filling in the Bernal stacked graphene layers. On the other hand, the dramatic impact of out-of-plane compression on the in-plane phonon frequencies in the A-A stacked graphene layers strongly indicates that the $s p^{2}$ electrons are also involved. The $s p^{2}$ electrons filling the low-density area will cause a decrease in the overlap of the electron orbitals of neighboring carbon atoms and, therefore, result in a decrease in the in-plane phonon frequency as the calculations show. When we compress the graphene bilayers further after the low-density area is filled, the in-plane phonon frequency will then increase, again just as the calculations show (change in 

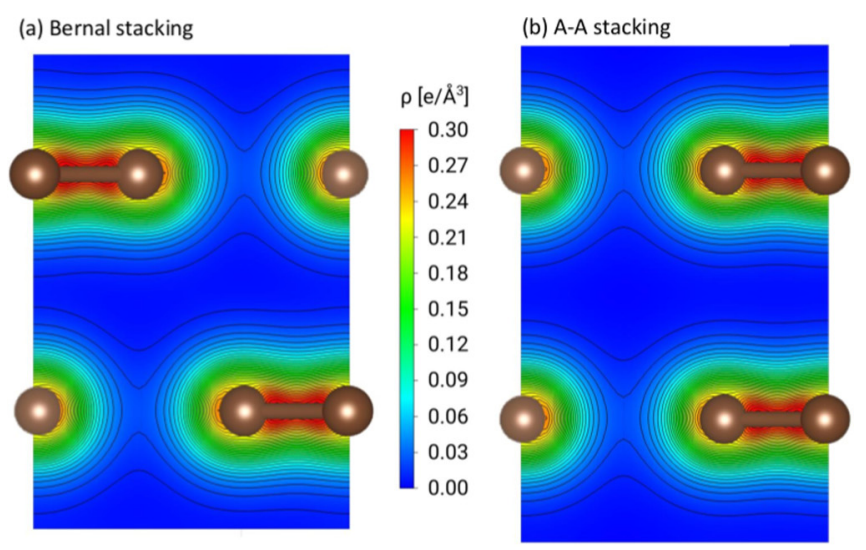

(c) Bernal stacking

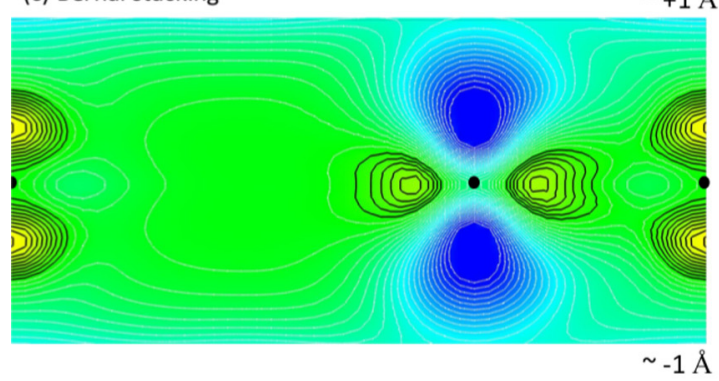

$\Delta \rho\left[\mathrm{e} / \AA^{3}\right]$

0.0020

0.0016

0.0012

0.0008

0.0004

0.0000

(d) A-A stacking

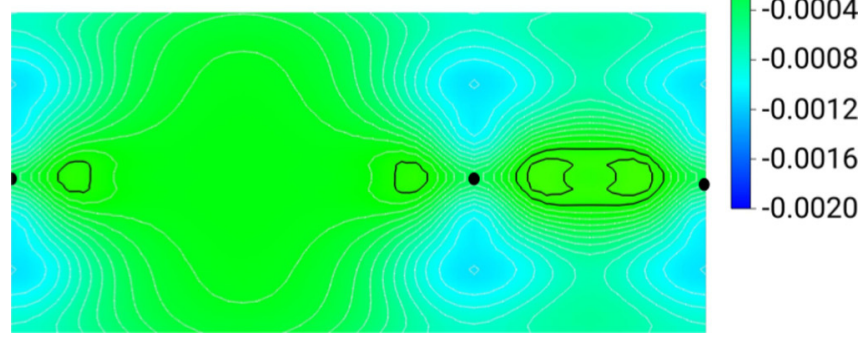

FIG. 5. The total valence charge density of bilayer graphene is plotted along the (110) plane for (a) Bernal and (b) A-A stackings. The color scale is labeled. Positions of atomic cores and $s p^{2}$ bonds are marked. The charge density of the $10 \%$ uniaxially compressed bilayer graphene is subtracted from that of the unstrained bilayer when we overlap their bottom layers, and it is then plotted for (c) Bernal and (d) A-A stackings. The contours are separated by $0.0001 e / \AA^{3}$. The (110) graphene plane through the carbon atoms is plotted in the middle of each plot. Atomic cores are labeled as black dots. We plot the charge density about $1 \AA$ above and below the graphene plane. Therefore, (c) and (d) are at over twice the magnification of (a) and (b).

the sign of the in-plane phonon shifts). Fahy et al. (1987) reported that the $s p^{2}$ to $s p^{3}$ transition of graphite is insensitive to the stacking order [33], indirectly supporting this interpretation. It is insensitive because the out-of-plane stiffness and the interlayer interaction for Bernal and A-A stackings are similar.

To illustrate this interpretation, we plot the valence charge density of bilayer graphene for A-A and Bernal stackings. We first plot the total valence charge density at equilibrium along the bilayer graphene (110) plane in Figs. 5(a) and 5(b) where two layers are shown and positions of atomic cores and $s p^{2}$ bonds are labeled. Comparing the charge density of the unstrained and compressed bilayers, we find that the difference in the charge density that causes a disruption in the $s p^{2}$ network is too small to be directly seen. Here, we overlap the bottom layers and plot the difference in charge density between the unstrained and $10 \%$ uniaxially compressed bilayer graphene for Bernal and A-A stackings in Figs. 5(c) and 5(d) [Figs. 5(c) and 5(d) are at over twice the magnification of Figs. 5(a) and 5(b)]. The blue color shows the increase in charge density under compression. The overlap of the $\pi$-electron orbitals is clearly seen-most obviously for Bernal stacking where the interlayer spacing is smaller (but only every other carbon has another directly above). We would like to point out that, under $10 \%$ compression, the integrated charge density between graphene bilayers is increased by about $10 \%$ as the absolute amount of electron charge density squeezed through the graphene planes is tiny. This increase mainly occurs for the $\pi$ orbitals. We now focus on the graphene plane where the $\mathrm{C}-\mathrm{C}$ bonding is changed. For Bernal stacking, the charge density is shown as green along the graphene plane in Fig. 5(c), indicating no change in the charge density, whereas in the A-A stacking, the $s p^{2}$ electrons "escape" out of the plane as the color turns yellowish in Fig. 5(d). This validates our proposed interpretation for the significant effect of the stacking order on the in-plane stiffness of graphene layers under uniaxial compression.

\section{CONCLUSIONS}

We used DFT to investigate the behavior of the $\pi$ electrons in graphene bilayers under compression along the $c$ axis and obtained the out-of-plane stiffness (along the $c$ axis), the overall interlayer interaction, and the in-plane phonon frequencies. There is a six times lower out-of-plane stiffness in bilayer graphene than in graphite. This is due to the asymmetric electron distribution on the two sides of each graphene layer in a bilayer where the relatively small amount of electron charge density (about $0.5 \%$ under $20 \%$ compression) compressed through the $s p^{2}$ network are relevant. The out-of-plane stiffness and the interlayer interaction, both of which are mainly determined by the $\pi$ electrons of the graphene bilayers, are very similar, but not identical, for both A-A and Bernal stackings. On the other hand, under out-of-plane compression, the shift of the in-plane phonons for A-A stacked graphene layers is significantly different from the shift for Bernal stacking. Both the small effects of the stacking order on the out-of-plane properties and the large effects on the in-plane properties are surprising. We propose the following interpretation: Under compression, the $\pi$-electron orbitals are considerably deformed, and some of their electrons fill the center areas of the carbon hexagons having low electron density. In addition, for A-A stacked graphene layers, the $s p^{2}$ electrons also contribute to the filling, inducing a softening of the $\mathrm{C}-\mathrm{C}$ bond when the compression starts. Our results exhibit the significant impact of the re-distribution of the $\pi$ and $s p^{2}$ orbitals on both the in-plane and the out-of-plane properties of graphene.

\section{ACKNOWLEDGMENTS}

D.H. and D.G. gratefully acknowledge financial support under the scope of the COMET Program within the K2 Center "Integrated Computational Material, Process and Product 
Engineering (IC-MPPE)" (Project No. 859480). This program was supported by the Austrian Federal Ministries for Transport, Innovation and Technology (BMVIT) and for Digital and Economic Affairs (BMDW), represented by the Austrian
Research Funding Association (FFG), and the federal states of Styria, Upper Austria, and Tyrol. The computational results presented have been achieved (in part) using the Vienna Scientific Cluster (VSC).
[1] C. Lee, X. Wei, J. W. Kysar, and J. Hone, Science 321, 385 (2008).

[2] M. Hanfland, H. Beister, and K. Syassen, Phys. Rev. B 39, 12598 (1989).

[3] Y. W. Sun, W. Liu, I. Hernandez, J. Gonzalez, F. Rodriguez, D. J. Dunstan, and C. J. Humphreys, Phys. Rev. Lett. 123, 135501 (2019).

[4] A. Bosak, M. Krisch, M. Mohr, J. Maultzsch, and C. Thomsen, Phys. Rev. B 75, 153408 (2007).

[5] C. Thomsen, S. Reich, and P. Ordejon, Phys. Rev. B 65, 073403 (2002).

[6] J. E. Proctor, E. Gregoryanz, K. S. Novoselov, M. Lotya, J. N. Coleman, and M. P. Halsall, Phys. Rev. B 80, 073408 (2009).

[7] M. Huang, H. Yan, C. Chen, D. Song, T. F. Heinz, and J. Hone, Proc. Natl. Acad. Sci. USA 106, 7304 (2009).

[8] T. M. G. Mohiuddin, A. Lombardo, R. R. Nair, A. Bonetti, G. Savini, R. Jalil, N. Bonini, D. M. Basko, C. Galiotis, N. Marzari, K. S. Novoselov, A. K. Geim, and A. C. Ferrari, Phys. Rev. B 79, 205433 (2009).

[9] Y. W. Sun, D. Holec, and D. J. Dunstan, Phys. Rev. B 92, 094108 (2015).

[10] M. Aoki and H. Amawashi, Solid State Commun. 142, 123 (2007).

[11] C. L. Lu, C. P. Chang, and M. F. Lin, Eur. Phys. J. B 60, 161 (2007).

[12] A. M. Popov, I. V. Lbedeva, A. A. Knizhnik, Y. E. Lozovik, B. V. Potapkin, N. A. Poklonski, A. I. Siahlo, and S. A. Vyrko, J. Chem. Phys. 139, 154705 (2013).

[13] J. W. Gonzalez, H. Santos, M. Pacheco, L. Chico, and L. Brey, Phys. Rev. B 81, 195406 (2010).

[14] I.-T. Lin, J.-M. Liu, K.-Y. Shi, P.-S. Tseng, K.-H. Wu, C.-W. Luo, and L.-J. Li, Phys. Rev. B 86, 235446 (2012).
[15] P. Lauffer, K. V. Emtsev, R. Graupner, T. Seyller, L. Ley, S. A. Reshanov, and H. B. Weber, Phys. Rev. B 77, 155426 (2008).

[16] Z. Liu, K. Suenaga, P. J. F. Harris, and S. Iijima, Phys. Rev. Lett. 102, 015501 (2009).

[17] P. L. de Andres, R. Ramirez, and J. A. Verges, Phys. Rev. B 77, 045403 (2008).

[18] P. Hohenberg and W. Kohn, Phys. Rev. 136, B864 (1964).

[19] W. Kohn and L. Sham, Phys. Rev. 140, A1133 (1965).

[20] G. Kresse and J. Furthmüller, Phys. Rev. B 54, 11169 (1996).

[21] J. P. Perdew, K. Burke, and M. Ernzerhof, Phys. Rev. Lett. 77, 3865 (1996).

[22] G. Kresse and D. Joubert, Phys. Rev. B 59, 1758 (1999).

[23] S. Grimme, J. Comput. Chem. 27, 1787 (2006).

[24] A. Togo, F. Oba, and I. Tanaka, Phys. Rev. B 78, 134106 (2008).

[25] K. Momma and F. Izumi, J. Appl. Cryst. 44, 1272 (2011).

[26] F. D. Murnaghan, Proc. Natl. Acad. Sci. USA 30, 244 (1944).

[27] P. Bundy, A. W. Bassett, M. S. Weathers, R. J. Hemley, H. K. Mao, and A. F. Goncharov, Carbon 34, 141 (1996).

[28] J. Proctor, Physics 12, 104 (2019).

[29] B. Alzyab, C. H. Perry, C. Zahopoulos, O. A. Pringle, and R. M. Nicklow, Phys. Rev. B 38, 1544 (1988).

[30] S. Reich and C. Thomsen, Philos. Trans. R. Soc. London, Ser. A 362, 2271 (2004).

[31] Y. W. Sun, D. Holec, Y. Xu, and D. J. Dunstan, J. Phys. Commun. 2, 045004 (2018).

[32] See Supplemental Material at http://link.aps.org/supplemental/ 10.1103/PhysRevB.101.125421 for the shifts of in-plane phonon frequencies with interlayer distance of the A-A and Bernal stacked bilayer graphene.

[33] S. Fahy, S. G. Louie, and M. L. Cohen, Phys. Rev. B 35, 7623 (1987). 